\title{
INVERSION OF RADON TRANSFORMS USING WAVELET TRANSFORMS GENERATED BY WAVELET MEASURES ${ }^{1}$
}

\author{
BORIS RUBIN
}

\begin{abstract}
The classical inversion formulae for the Radon transform $R$ on $\mathrm{R}^{n}$ involve the dual transform $R^{\#}$ and the operator $D^{n-1}=(-\Delta)^{(n-1) / 2}$ which represents the positive power of the Laplacian. It is shown that wavelet type representations of $D^{n-1}$, generated by finite measures with a certain number of vanishing moments, lead to explicit inversion formulae for $R f, f \in L^{p}\left(\mathrm{R}^{n}\right)$, and enable one to characterize the range $R\left(L^{p}\left(\mathrm{R}^{n}\right)\right)$. The same method can be applied to explicit inversion and characterization of Radon transforms of finite Borel measures.
\end{abstract}

\section{Introduction}

The Radon transform of sufficiently nice function $f$ on $\mathrm{R}^{n}$ is defined by

$$
\left(R_{\theta} f\right)(s) \equiv(R f)(\theta, s)=\int_{\theta^{\perp}} f(s \theta+u) d u, \quad(\theta, s) \in \tilde{\mathrm{R}}^{n}=\Sigma_{n-1} \times \mathrm{R},
$$

where $\Sigma_{n-1}$ is the unit sphere in $\mathrm{R}^{n}, d u$ stands for the euclidean measure on the subspace $\theta^{\perp}$ orthogonal to $\theta$. Among the basic problems related to (1.1) are explicit inversion of the operator $R$ and a characterization of its range. The relations

$$
f=\lambda_{n} R^{\#}\left(-\Delta_{1}\right)^{(n-1) / 2} \varphi \text { and } f=\lambda_{n}\left(-\Delta_{n}\right)^{(n-1) / 2} R^{\#} \varphi, \quad \varphi=R f,
$$

$\lambda_{n}=(2 \pi)^{1-n} / 2$ (see e.g. [5]), involving the dual transform

$$
\left(R^{\#} \varphi\right)(x)=\int_{\Sigma_{n-1}} \varphi(\theta,\langle x, \theta\rangle) d \theta, \quad x \in \mathrm{R}^{n},
$$

and the $(n-1) / 2$ th power of the Laplacian (in one and $n$ dimensions respectively) are usually employed for solution of these problems. In spite of the elegance and simplicity of (1.2), the practical implementation of these

\footnotetext{
* Partially sponsored by the Edmund Landau Center for research in Mathematical analysis, supported by the Minerva Foundation (Germany).

Received January 9, 1997; in revised form March 11, 1997.
} 
formulae entails difficulties connected with the realization of powers of the Laplacian. These difficulties increase when dealing with nonsmooth functions or measures, the differentiation of which can be performed only in the distribution sense. Additional difficulties arise in the case of $n$ even, when the operator $(-\Delta)^{(n-1) / 2}$ is not local. In order to reduce these difficulties, in [1, $3,8,14]$ it was suggested to employ continuous wavelet transforms.

In the present paper we show that wavelet constructions of the inverse Radon transform can be obtained directly from (1.2) if one replaces the powers of the Laplacians by their wavelet representations (see [7, 9]) generated by suitable wavelet measures. For example, the first formula from (1.2) gives rise to the following statement.

Theorem 1.1. Assume that $\mu$ is a finite Borel measure on $\mathrm{R}$ satisfying the following conditions:

$\int_{|s|>1}|s|^{\beta} d|\mu|(s)<\infty$ for some $\beta>n-1 ; \int_{-\infty}^{\infty} s^{j} d \mu(s)=0$ for all $j=0,2, \ldots, 2[(n-1) / 2]$

where $[(n-1) / 2]$ designates the integer part of $(n-1) / 2$.

(i) Let $\varphi=R f$, $f \in L^{p}\left(\mathrm{R}^{n}\right), 1 \leq p<n /(n-1)$. Denote

$\left(T_{\varepsilon} \varphi\right)(x)=\int_{\varepsilon}^{\infty} R^{\#}\left(\varphi * \mu_{t}\right)(x) \frac{d t}{t^{n}}, \varepsilon>0 ;\left(\varphi * \mu_{t}\right)(\theta, s)=\int_{-\infty}^{\infty} \varphi(\theta, s-t \eta) d \mu(\eta)$.

Then

$$
\int_{0}^{\infty} R^{\#}\left(\varphi * \mu_{t}\right) \frac{d t}{t^{n}} \equiv \lim _{\varepsilon \rightarrow 0}\left(T_{\varepsilon} \varphi\right)(x)=c_{\mu} f
$$

the limit being interpreted in the $L^{p}$-norm and in the a.e. sense,

$$
c_{\mu}=\left\{\begin{array}{l}
\frac{\pi^{n+1 / 2}(-1)^{n / 2}}{\Gamma(n / 2) \Gamma((n+1) / 2)} \int_{-\infty}^{\infty}|s|^{n-1} d \mu(s) \quad \text { if } n \text { is even, } \\
\frac{2 \pi^{n-1 / 2}(-1)^{(n+1) / 2}}{\Gamma(n / 2) \Gamma((n+1) / 2)} \int_{-\infty}^{\infty}|s|^{n-1} \log |s| d \mu(s) \quad \text { if } n \text { is odd } .
\end{array}\right.
$$

(ii) Let

$$
\varphi \in L^{1, r}\left(\tilde{\mathbf{R}}^{n}\right)=\left\{\varphi(\theta, s):\|\varphi\|_{1, r}=\int_{\Sigma_{n-1}}\left[\int_{-\infty}^{\infty}|\varphi(\theta, s)|^{r} d s\right]^{1 / r} d \theta<\infty\right\},
$$

$r^{-1}=n p^{-1}-n+1,1 \leq p<n /(n-1)$. If $c_{\mu} \neq 0$, then the following statements are equivalent: (a) $\varphi \in R\left(L^{p}\left(\mathrm{R}^{n}\right)\right)$; (b) $T_{\varepsilon} \varphi$ converges in the $L^{p}\left(\mathrm{R}^{n}\right)$-norm as $\varepsilon \rightarrow 0$. If $1<p<n /(n-1)$, then (a) and (b) are equivalent to (c) $\sup _{\varepsilon>0}\left\|T_{\varepsilon} \varphi\right\|_{p}<\infty$. 
REMARK 1.2. The relation (1.5) also holds provided $\varphi=R f, f \in L^{q}\left(\mathrm{R}^{n}\right)$ $\cap L^{p}\left(\mathrm{R}^{n}\right)$, where $1 \leq q<n /(n-1), 1 \leq p \leq \infty \quad\left(L^{\infty}\right.$ is identified with the space $C_{0}$ of continuous functions vanishing at infinity), and can be written in the form

$$
c_{\mu} f=\lim _{\varepsilon \rightarrow 0} \int_{|y|>\varepsilon} \frac{d y}{|y|^{2 n-1}} \int_{-\infty}^{\infty} \varphi\left(y^{\prime},\left\langle x, y^{\prime}\right\rangle-s|y|\right) d \mu(s), \quad y^{\prime}=\frac{y}{|y|} .
$$

Let us give some examples of measures which satisfy the conditions of Theorem 1.1.

EXAMPLE 1.3. Let $\delta_{k}=\delta_{k}(s)$ be the unit Dirac mass at the point $s=k$,

$$
\mu=\sum_{k=0}^{l}(-1)^{k}\left(\begin{array}{l}
l \\
k
\end{array}\right) \delta_{k}, \quad l>2[(n-1) / 2] .
$$

The function $A_{l}(\alpha)=\sum_{k=0}^{l}(-1)^{k-1}\left(\begin{array}{l}l \\ k\end{array}\right) k^{\alpha}, \alpha>0, l \in \mathrm{N}$, vanishes at the points $\alpha=1,2, \ldots, l-1$ and nowhere else on $\mathbf{R}^{+}$(see [11], p. 116-117, 506-507). At these points $A_{l}(\alpha)$ has a simple zero. Thus, $\int_{-\infty}^{\infty} s^{j} d \mu(s)=A_{l}(j)=0$ for $j=0,1, \ldots, l-1$ provided $l>2[(n-1) / 2]$. By $(1.5),(1.8)$, for $\varphi=R f$ this yields

$$
\begin{aligned}
& f=\frac{1}{\varkappa_{n}} \int_{0}^{\infty} \frac{d t}{t^{n}} \int_{\Sigma_{n-1}}\left[\sum_{k=0}^{l}(-1)^{k}\left(\begin{array}{l}
l \\
k
\end{array}\right) \varphi(\theta,\langle x, \theta\rangle-k t)\right] d \theta \\
& =\frac{1}{\varkappa_{n}} \int_{\mathrm{R}^{n}}\left[\sum_{k=0}^{l}(-1)^{k}\left(\begin{array}{l}
l \\
k
\end{array}\right) \varphi\left(y^{\prime},\left\langle x, y^{\prime}\right\rangle-k|y|\right)\right] \frac{d y}{|y|^{2 n-1}}
\end{aligned}
$$

where

$$
\varkappa_{n}=\left\{\begin{array}{l}
\frac{\pi^{n+1 / 2}(-1)^{(n-2) / 2}}{\Gamma(n / 2) \Gamma((n+1) / 2)}, \quad l=n-1, \quad \text { if } n \text { is even, } \\
\frac{2 \pi^{n-1 / 2}(-1)^{(n-1) / 2}}{\Gamma(n / 2) \Gamma((n+1) / 2)}\left[\frac{d}{d \alpha} A_{l}(\alpha)\right]_{\alpha=1-n}, \quad l>n-1, \quad \text { if } n \text { is odd } .
\end{array}\right.
$$

Note that $x_{n} \neq 0$ under the choice of $l$ stated in (1.12). The expressions (1.10), (1.11) are hypersingular integrals of the Marchaud type. Similar integrals are widely used for characterization of functions of the fractional smoothness and for explicit inversion of integral operators of the potential type (see $[7,11]$ and references therein).

An example of a measure $\mu$, generalizing (1.9) and satisfying the conditions of Theorem 1.1 with $c_{\mu} \neq 0$, is given by the determinant 


$$
\mu=\left|\begin{array}{ccccc}
\delta_{\lambda_{0}} & 1 & \lambda_{0} & \cdots & \lambda_{0}^{\ell-1} \\
\delta_{\lambda_{1}} & 1 & \lambda_{1} & \cdots & \lambda_{1}^{\ell-1} \\
\cdots & \cdots & \cdots & \cdots & \cdots \\
\delta_{\lambda_{\ell}} & 1 & \lambda_{\ell} & \cdots & \lambda_{\ell}^{\ell-1}
\end{array}\right|
$$

where $\delta_{\lambda_{k}}$ are Dirac masses at arbitrary points $\lambda_{k}$ such that $0 \leq \lambda_{0}<\lambda_{1}$ $<\ldots<\lambda_{l}$ (see [7], Section 10). A trivial example of the absolutely continuous measure $\mu$, satisfying required conditions, can be obtained, e.g., by differentiating the function $\exp \left(-x^{2}\right)$. More interesting example (cf. [7], p.140), which serves all $n \geq 2$, is given by $d \mu(s)=w(s) d s$ where

(1.13) $w(s) \equiv 0$ for $s \leq 0$ and $w(s)=\operatorname{sexp}\left(-\log ^{2} s\right) \sin (\pi \log s)$ for $s>0$.

This function is infinitely smooth and decreases rapidly as $s \rightarrow 0, \infty$. The Mellin transformation of $w$ has the form $\int_{0}^{\infty} s^{z-1} w(s) d s=\pi^{1 / 2} \exp \left(\left((z+1)^{2}\right.\right.$ $-\pi) / 4) \cos (\pi z / 2)$ and therefore all even moments of $w$ are zero. Furthermore, by (1.6),

$$
c_{\mu}=\frac{\pi^{n+1}}{\Gamma(n / 2) \Gamma((n+1) / 2)} \exp \left(\frac{(n+1)^{2}-\pi}{4}\right) \neq 0 \quad \text { for all } n \geq 2 .
$$

In order to state the result, corresponding to the second equality from (1.2), given a finite measure $\nu$ on $\mathrm{R}^{n}$, we denote

$$
B_{\varepsilon} \varphi=\int_{\mathrm{SO}(n)} d \gamma \int_{\varepsilon}^{\infty}\left(R^{\#} \varphi * \nu_{\gamma, t}\right) \frac{d t}{t^{n}}, \quad \varepsilon>0,
$$

where $\left(R^{\#} \varphi * \nu_{\gamma, t}\right)(x)=\int_{\mathrm{R}^{n}}\left(R^{\#} \varphi\right)(x-t \gamma y) d \nu(y)$.

THEOREM 1.4. Let $1 \leq p<n /(n-1)$ and assume that $\nu$ satisfies the following conditions:

$$
\begin{aligned}
& \int_{|x|>1}|x|^{\beta} d|\nu|(x)<\infty \quad \text { for some } \beta>n-1 \\
& \int_{\mathrm{R}^{n}} x^{j} d \nu(x)=0 \quad \text { for all }|j|=0,2,4, \ldots, 2[(n-1) / 2] .
\end{aligned}
$$

(i) If $\varphi=R f$, $f \in L^{p}\left(\mathrm{R}^{n}\right)$, then

$$
\int_{S O(n)} d \gamma \int_{0}^{\infty}\left(R^{\#} \varphi * \nu_{\gamma, t}\right) \frac{d t}{t^{n}} \equiv \lim _{\varepsilon \rightarrow 0} B_{\varepsilon} \varphi=d_{\nu} f
$$

where the limit is interpreted in the $L^{p}$-norm and in the a.e. sense,

$$
d_{\nu}=\left\{\begin{array}{l}
\frac{\pi^{n}(-1)^{n / 2} \Gamma(n / 2)}{\Gamma(n-1 / 2) \Gamma((n+1) / 2)} \int_{\mathrm{R}^{n}}|y|^{n-1} d \nu(y) \quad \text { if } n \text { is even } \\
\frac{2 \pi^{n-1}(-1)^{(n+1) / 2} \Gamma(n / 2)}{\Gamma(n-1 / 2) \Gamma((n+1) / 2)} \int_{\mathrm{R}^{n}}|y|^{n-1} \log |y| d \nu(y) \quad \text { if } n \text { is odd }
\end{array}\right.
$$


(ii) Let $\varphi \in L^{1, r}\left(\tilde{\mathrm{R}}^{n}\right), r^{-1}=n p^{-1}-n+1$. If $d_{\nu} \neq 0$, then the following statements are equivalent: (a) $\varphi \in R\left(L^{p}\left(\mathrm{R}^{n}\right)\right)$; (b) $B_{\varepsilon} \varphi$ converges in the $L^{p}\left(\mathrm{R}^{n}\right)$ norm as $\varepsilon \rightarrow 0$. If $1<p<n /(n-1)$, then (a) and (b) are equivalent to (c) $\sup _{\varepsilon>0}\left\|B_{\varepsilon} \varphi\right\|_{p}<\infty$.

The relation (1.15) also holds provided $\varphi=R f, f \in L^{q}\left(\mathrm{R}^{n}\right) \cap L^{p}\left(\mathrm{R}^{n}\right)$, where $1 \leq q<n /(n-1), 1 \leq p \leq \infty$ (cf. Remark 1.2). If $\nu$ is radial, then (1.15) reads

$$
\int_{0}^{\infty}\left(R^{\#} \varphi * \nu_{t}\right) \frac{d t}{t^{n}}=d_{\nu} f, \quad \varphi=R f .
$$

Various examples of measures satisfying conditions of Theorem 1.4 can be found in [9]. Formulae (1.5), (1.15) contain those from [3, 8] as particular cases. If $\mu$ and $\nu$ are absolutely continuous, then the convolutions $\varphi * \mu_{t}$ and $R^{\#} \varphi * \nu_{\gamma, t}$ are just continuous wavelet transforms of $\varphi$ and $R^{\#} \varphi$ respectively. If $\nu=u * v$, then (1.15) yields the wavelet decomposition of $f$ with the analysing wavelet $u$ and the reconstructing wavelet $v$ (or vice versa). The integration over the rotation group $S O(n)$ can be replaced by the integration over the $(n-1)$-dimensional unit sphere (see [9]). The conditions for wavelet measures can be reformulated in terms of their Fourier transforms (in the framework of the corresponding $L^{2}$-theory). The interested reader is addressed to [10] for the details.

The paper is organized as follows. Section 2 contains basic definitions and some auxiliary facts. In Sections 3 and 4 we prove Theorem 1.1 and Theorem 1.4 respectively. The analogous statements are established for the case when $f$ is replaced by a finite Borel measure.

Acknowledgement. The author is grateful to Prof. Robert S. Strichartz for valuable remarks, which led to improvement of the text of the paper.

Notation. For $x=\left(x_{1}, \ldots, x_{n}\right) \in \mathbf{R}^{n}$ and $y=\left(y_{1}, \ldots, y_{n}\right) \in \mathbf{R}^{n}$ we write $\langle x, y\rangle=x_{1} y_{1}+\ldots+x_{n} y_{n}$. Let $e_{1}=(1,0, \ldots, 0), \quad \Sigma_{n-1}=\left\{x \in \mathrm{R}^{n}:|x|=1\right\}$, $\left|\Sigma_{n-1}\right|=2 \pi^{n / 2} / \Gamma(n / 2) ;[a]$ is the integer part of the real number $a$. Given a function $k(x)$ on $\mathrm{R}^{n}$ and $\varepsilon>0$ (instead of $\varepsilon$ there may be $t, \rho$ or another letter) we denote $k_{\varepsilon}(x)=\varepsilon^{-n} k(x / \varepsilon)$. The notation $C\left(\mathrm{R}^{n}\right), C^{\infty}\left(\mathrm{R}^{n}\right), L^{p}\left(\mathrm{R}^{n}\right)$ is standard; $C_{0}\left(\mathrm{R}^{n}\right)=\left\{f \in C\left(\mathrm{R}^{n}\right): \lim _{|x| \rightarrow \infty} f(x)=0\right\} ; C_{c}^{\infty}\left(\mathrm{R}^{n}\right)$ is the space of compactly supported infinitely differentiable functions; $\mathcal{S}\left(\mathrm{R}^{n}\right)$ is the Schwartz space of rapidly decreasing $C^{\infty}$-functions with a standard topology. The Fourier transform and its inverse are defined by

$\hat{f}(\xi)=(F f)(\xi)=\int_{\mathrm{R}^{n}} f(x) e^{i\langle x, \xi\rangle} d x, \quad \breve{f}(x)=\left(F^{-1} f\right)(x)=(2 \pi)^{-n} \int_{\mathrm{R}^{n}} f(\xi) e^{-i\langle x, \xi\rangle} d \xi$.

We denote by $\Phi\left(\mathrm{R}^{n}\right)$ the subspace of $\mathcal{S}\left(\mathrm{R}^{n}\right)$, which consists of functions or- 
thogonal to all polynomials; $\Psi=F \Phi\left(\mathrm{R}^{n}\right) . \mathcal{M}\left(\mathrm{R}^{n}\right)$ is the Banach space of finite Borel measures $\mu$ on $\mathrm{R}^{n}$ with the norm $\|\mu\|$ designating the total variation of $|\mu|$. The letter $c$ is used for constants which can be different in each occasion.

\section{Preliminaries}

We recall some properties of the Radon transform (1.1). If $f \in L^{1}\left(\mathrm{R}^{n}\right)$, then $R_{\theta} f \in L^{1}(\mathrm{R})$ and by the Fubini theorem $\left\|R_{\theta} f\right\|_{L^{1}(\mathrm{R})} \leq\|f\|_{L^{1}\left(\mathrm{R}^{n}\right)}$ for each $\theta \in \Sigma_{n-1}$. If $f \in L^{p}\left(\mathrm{R}^{n}\right), 1 \leq p<n /(n-1)$, then $(R f)(\theta, s)$ is locally integrable on $\tilde{\mathrm{R}}^{n}$. The restriction $p<n /(n-1)$ is essential. For example, the function $\stackrel{\circ}{f}(x)=(2+|x|)^{-n / p}(\log (2+|x|))^{-1}$ belongs to $L^{p}\left(\mathrm{R}^{n}\right), p \geq n /(n-1)$, and $\left(R_{\theta} f\right) \quad(s) \equiv \infty \quad \forall \theta \in \Sigma_{n-1} \quad$ (see [13]). If $1 \leq p<n /(n-1) \quad$ and $r^{-1}=n p^{-1}-n+1$, then $R$ is a linear bounded operator from $L^{p}\left(\mathrm{R}^{n}\right)$ into the space $L^{1, r}\left(\tilde{\mathrm{R}}^{n}\right)$ defined in (1.7) (see [6]).

The Radon transform $R_{\theta} \lambda$ of the Borel measure $\lambda$ on $\mathrm{R}^{n}$ can be defined as the image of $\lambda$ under the mapping $r_{\theta}^{-1} E_{\theta}$, where $E_{\theta}$ is the orthogonal projection onto the line $l_{\theta}=\{s \theta:-\infty<s<\infty\}$ and $r_{\theta}$ is an arbitrary rotation such that $r_{\theta} e_{1}=\theta$. This means that $R_{\theta} \lambda$ is the Borel measure on $\mathbf{R}$ such that $\left(R_{\theta} \lambda\right)(\Omega)=\lambda\left(E_{\theta}^{-1} r_{\theta} \Omega\right)=\lambda\left(\theta^{\perp} \times r_{\theta} \Omega\right), \quad \Omega \subset \mathbf{R}$. The definition of $R_{\theta} \lambda$ does not depend on the choice of $r_{\theta}$ and corresponds to (1.1). Clearly, if $\lambda$ is finite on $\mathrm{R}^{n}$, then $R_{\theta} \lambda$ is finite on $\mathrm{R}$ for each $\theta \in \Sigma_{n-1}$. The idea of the above definition was borrowed from the more general consideration in [4].

Lemma 2.1. If $\lambda$ is a Borel measure on $\mathrm{R}^{n}$ and $\varphi$ is a Borel function on $\mathrm{R}$, then

$$
\int_{-\infty}^{\infty} \varphi(s) d\left(R_{\theta} \lambda\right)(s)=\int_{\mathrm{R}^{n}} \varphi\left(r_{\theta}^{-1} E_{\theta} x\right) d \lambda(x)=\int_{\mathrm{R}^{n}} \varphi(\langle x, \theta\rangle) d \lambda(x) .
$$

This statement follows from [4] (Theorem 1.19). Assuming $\varphi(s) \equiv \varphi(\theta, s)$ and integrating the above equality over $\Sigma_{n-1}$ we get

$$
\int_{\Sigma_{n-1}} d \theta \int_{\mathrm{R}} \varphi(\theta, s) d\left(R_{\theta} \lambda\right)(s)=\int_{\mathrm{R}^{n}} d \lambda(x) \int_{\Sigma_{n-1}} \varphi(\theta,\langle x, \theta\rangle) d \theta .
$$

Given Borel measures $\nu, \mu$ and functions $\varphi, \psi$ on $\tilde{\mathrm{R}}^{n}$ and $\mathbf{R}^{n}$ respectively, we denote

$$
(\nu, \varphi)^{\sim}=\int_{\tilde{\mathrm{R}}^{n}} \overline{\varphi(\theta, s)} d \nu(\theta, s), \quad(\lambda, \psi)=\int_{\mathrm{R}^{n}} \overline{\psi(x)} d \lambda(x) .
$$

Let $R \lambda$ be a measure on $\tilde{\mathrm{R}}^{n}$ defined by $d(R \lambda)(\theta, s)=d \theta d\left(R_{\theta} \lambda\right)(s)$. Then (2.1) reads

$$
(R \lambda, \varphi)^{\sim}=\left(\lambda, R^{\#} \varphi\right)
$$


$R^{\#} \varphi$ being the dual Radon transform (1.3). In particular, if $d \lambda(x)=f(x) d x$, then

$$
(R f, \varphi)^{\sim}=\left(f, R^{\#} \varphi\right) .
$$

We remark that $R \lambda \equiv R_{\theta} \lambda$ is an even finite measure on $\tilde{\mathrm{R}}^{n}$. If $\lambda$ is absolutely continuous, i.e. $d \lambda(x)=f(x) d x$, then $d(R \lambda)(\theta, s)=(R f)(\theta, s) d \theta d s$.

Let $\mathcal{S}\left(\tilde{\mathrm{R}}^{n}\right)$ be the space of rapidly decreasing even smooth functions $g(\theta, s)$ on $\tilde{\mathrm{R}}^{n}$ with the topology generated by the sequence of norms

$$
\|g\|_{m}=\sup _{|\alpha|+j \leq m} \sup _{\theta, s}(1+|s|)^{m}\left|\partial_{\theta}^{\alpha} \partial_{s}^{j} g(\theta, s)\right|, \quad m=0,1, \ldots,
$$

where

$$
\partial_{\theta}^{\alpha} g(\theta, s)=\left[\frac{\partial^{|\alpha|} g(x /|x|, s)}{\partial x_{1}^{\alpha_{1}} \ldots \partial x_{n}^{\alpha_{n}}}\right]_{x=\theta}, \quad \alpha=\left(\alpha_{1}, \ldots, \alpha_{n}\right) \in Z_{+}^{n} .
$$

Following [12, 2], we consider the subspaces of $\mathcal{S}\left(\tilde{\mathrm{R}}^{n}\right)$ defined by

$\Psi\left(\tilde{\mathbf{R}}^{n}\right)=\left\{\psi(\theta, \eta) \in \mathcal{S}\left(\tilde{\mathbf{R}}^{n}\right):\left(\partial_{\theta}^{\alpha} \partial_{\eta}^{j} \psi\right)(\theta, 0)=0\right.$, for all $\left.\alpha \in Z_{+}^{n}, j \in Z_{+}, \theta \in \Sigma_{n-1}\right\}$, $\Phi\left(\tilde{\mathrm{R}}^{n}\right)=F \Psi\left(\tilde{\mathrm{R}}^{n}\right)=\left\{\varphi(\theta, s) \in \mathcal{S}\left(\tilde{\mathbf{R}}^{n}\right):\right.$

$$
\left.\int_{-\infty}^{\infty} s^{j} \partial_{\theta}^{\alpha} \partial_{s}^{k} g(\theta, s) d s=0, \text { for all } j \in Z_{+}, \alpha \in Z_{+}^{n}, k \in Z_{+}, \theta \in \Sigma_{n-1}\right\}
$$

(here $F$ denotes the Fourier transform in the second variable).

Lemma $2.2([12,2,10])$. The operator $R\left(R^{\#}\right)$ acts as the isomorphism from $\Phi\left(\mathbf{R}^{n}\right)$ onto $\Phi\left(\tilde{\mathrm{R}}^{n}\right)\left(\right.$ from $\Phi\left(\tilde{\mathrm{R}}^{n}\right)$ onto $\left.\Phi\left(\mathbf{R}^{n}\right)\right)$.

We denote by $\mathcal{S}^{\prime}\left(\mathrm{R}^{n}\right), \Phi^{\prime}\left(\mathrm{R}^{n}\right), \mathcal{S}^{\prime}\left(\tilde{\mathrm{R}}^{n}\right)$ and $\Phi^{\prime}\left(\tilde{\mathrm{R}}^{n}\right)$ the duals of $\mathcal{S}\left(\mathrm{R}^{n}\right), \Phi\left(\mathrm{R}^{n}\right)$, $\mathcal{S}\left(\tilde{\mathbf{R}}^{n}\right)$ and $\Phi\left(\tilde{\mathbf{R}}^{n}\right)$ respectively. Clearly, $L^{p}\left(\mathbf{R}^{n}\right) \subset \Phi^{\prime}\left(\mathbf{R}^{n}\right)$ and $L^{1, r}\left(\tilde{\mathbf{R}}^{n}\right) \subset \Phi^{\prime}\left(\tilde{\mathbf{R}}^{n}\right)$ for $1 \leq p, r \leq \infty$.

Lemma 2.3. (i) Let $f \in L^{p}\left(\mathrm{R}^{n}\right), 1 \leq p<\infty$, and $g \in L^{q}\left(\mathrm{R}^{n}\right), 1 \leq q<\infty$. If $f=g$ in the $\Phi^{\prime}\left(\mathbf{R}^{n}\right)$-sense, then $f=g$ a.e. on $\mathbf{R}^{n}$.

(ii) Let $\mu \in \mathcal{M}\left(\mathbf{R}^{n}\right)$. If $\mu=0$ in the $\Phi^{\prime}\left(\mathbf{R}^{n}\right)$-sense, then $(\mu, u)=0$ for each $u \in C_{0}\left(\mathrm{R}^{n}\right)$.

Proof. The proof of the first statement can be found in [7]. In order to check (ii) we pick up an arbitrary bump function $a(x) \in \mathcal{S}\left(\mathrm{R}^{n}\right)$ with $\int a=1$ and consider the convolution $a_{\varepsilon} * \mu$ with $a_{\varepsilon}(x)=\varepsilon^{-n} a(x / \varepsilon), \varepsilon>0$. Clearly, $a_{\varepsilon} * \mu \in L^{1}\left(\mathrm{R}^{n}\right)$, and for any $v \in \Phi\left(\mathrm{R}^{n}\right)$ we have $a_{\varepsilon} * v \in \Phi\left(\mathrm{R}^{n}\right)$. It follows that $\left(a_{\varepsilon} * \mu, v\right)=\left(\mu, a_{\varepsilon} * v\right)=0$ and therefore, according to (i), $a_{\varepsilon} * \mu \stackrel{\text { a.e. }}{=} 0$. Hence for each $u \in C_{0}\left(\mathrm{R}^{n}\right)$ we get $0=\left(a_{\varepsilon} * \mu, u\right)=\lim _{\varepsilon \rightarrow 0}\left(a_{\varepsilon} * \mu, u\right)=\lim _{\varepsilon \rightarrow 0}\left(\mu, a_{\varepsilon} * u\right)$ $=(\mu, u)$, which was required. 
Our next goal is to prove an analogue of Lemma 2.3 for $\tilde{\mathrm{R}}^{n}$. Let $\mathcal{M}\left(\tilde{\mathrm{R}}^{n}\right)$ be the Banach space of all finite Borel measures on $\tilde{\mathbf{R}}^{n} ; C_{0}\left(\tilde{\mathbf{R}}^{n}\right)=\left\{v(\theta, s) \in C\left(\tilde{\mathbf{R}}^{n}\right)\right.$ : $\lim _{s \rightarrow \pm \infty} v(\theta, s)=0$ for each $\left.\theta \in \Sigma_{n-1}\right\}$. We denote by $\left\{Y_{j, k}(\theta)\right\}$ the orthonormal basis of spherical harmonics on $\Sigma_{n-1}$. Here $j \in Z_{+}$and $k=1,2, \ldots, d_{n}(j)$, where $d_{n}(j)$ is the dimension of the subspace of spherical harmonics of degree $j$. Given a function $g \in L^{1}\left(\Sigma_{n-1}\right)$, the Fourier-Laplace coefficients of $g$ are defined by $g_{j, k}=\int_{\Sigma_{n-1}} g(\theta) Y_{j, k}(\theta) d \theta$.

Lemma 2.4. (i) Let $\varphi \in L^{1, r}\left(\tilde{\mathbf{R}}^{n}\right), \psi \in L^{1, \rho}\left(\tilde{\mathbf{R}}^{n}\right), 1 \leq r, \rho<\infty$. If $\varphi=\psi$ in the $\Phi^{\prime}\left(\tilde{\mathbf{R}}^{n}\right)$-sense, then $\varphi=\psi$ a.e. on $\tilde{\mathbf{R}}^{n}$.

(ii) Let $\mu \in \mathcal{M}\left(\tilde{\mathbf{R}}^{n}\right)$. If $\mu=0$ in the $\Phi^{\prime}\left(\tilde{\mathbf{R}}^{n}\right)$-sense, then $(\mu, v)=0$ for each $v \in C_{0}\left(\tilde{\mathrm{R}}^{n}\right)$.

Proof. Consider the Fourier-Laplace coefficients of $\varphi(\theta, s)$ and $\psi(\theta, s)$ in the $\theta$-variable. Clearly, $\varphi_{j, k}(s) \in L^{r}(\mathbf{R})$ and $\psi_{j, k}(s) \in L^{q}(\mathbf{R})$. Moreover, $\varphi_{j, k}=\psi_{j, k}$ in the $\Phi^{\prime}(\mathrm{R})$-sense. Indeed, let $u \in \Phi(\mathrm{R}), u_{j, k}(\theta, s)=u(s) Y_{j, k}(\theta)$. By taking into account the evenness of $\varphi$ we have $\left(\varphi_{j, k}, u\right)=\left(\varphi, u_{j, k}\right)^{\sim}=([\varphi(\theta, s)+$ $\left.\varphi(-\theta,-s)] / 2, u_{j, k}(\theta, s)\right)^{\sim}=\left(\varphi, u_{j, k}^{+}\right)^{\sim}$, where $u_{j, k}^{+}(\theta, s)=\left[u_{j, k}(\theta, s)+u_{j, k}(-\theta,-s)\right] / 2$ $\in \Phi\left(\tilde{\mathbf{R}}^{n}\right)$. Since $\varphi$ and $\psi$ coincide as the $\Phi^{\prime}\left(\tilde{\mathbf{R}}^{n}\right)$-distributions, then $\left(\varphi_{j, k}, u\right)$ $=\left(\varphi, u_{j, k}^{+}\right)^{\sim}=\left(\psi, u_{j, k}^{+}\right)^{\sim}=\left(\psi_{j, k}, u\right) \quad \forall u \in \Phi(\mathrm{R})$. By Lemma 2.3 it follows that $\varphi_{j, k}=\psi_{j, k}$ a.e. on $\mathbf{R}$, and therefore $\varphi=\psi$ a.e. on $\tilde{\mathbf{R}}^{n}$.

In order to prove (ii) we take a bump function $a \in \mathcal{S}(\mathrm{R})$ with $\int a=1$ and the Poisson kernel $\mathcal{P}_{t}(\theta, \sigma)=c_{n}\left(1-t^{2}\right) /|\theta-t \sigma|^{n}, \quad t \in[0,1), \quad c_{n}=1 /\left|\Sigma_{n-1}\right|$. Let $a_{\varepsilon}(s)=\varepsilon^{-1} a(s / \varepsilon)$. The function $\left(A_{\varepsilon, t} \mu\right)(s, \theta)=\int_{\tilde{\mathrm{R}}^{n}} a_{\varepsilon}(s-\eta) \mathcal{P}_{t}(\theta, \sigma) d \mu(\sigma, \eta)$, $\varepsilon \in(0,1)$, belongs to $L^{1,1}\left(\tilde{\mathrm{R}}^{n}\right)$, and for each $\omega \in \Phi\left(\tilde{\mathrm{R}}^{n}\right)$ we have $\left(A_{\varepsilon, t} \omega\right)$ $(s, \theta) \in \Phi\left(\tilde{\mathbf{R}}^{n}\right)$. Since $\left(A_{\varepsilon, t} \mu, \omega\right)=\left(\mu, A_{\varepsilon, t} \omega\right)=0 \forall \omega \in \Phi\left(\tilde{\mathbf{R}}^{n}\right)$, then by (i), $A_{\varepsilon, t} \mu=0$ a.e. on $\tilde{\mathrm{R}}^{n}$. By taking into account that $\int_{\Sigma_{n-1}} \mathcal{P}_{t}(\theta, \sigma) d \sigma \equiv 1$, for any $v \in C_{0}\left(\tilde{\mathrm{R}}^{n}\right)$ we get $0=\left(A_{\varepsilon, t} \mu, v\right)=\lim _{\substack{\varepsilon \rightarrow 0 \\ t \rightarrow 1}}\left(\mu, A_{\varepsilon, t} v\right)=(\mu, v)$.

Let us recall some facts related to Riesz potentials (see, e.g., [7, 11]). The Riesz potential $I^{\alpha} f, \operatorname{Re} \alpha>0$, on $\mathrm{R}^{n}$ is defined as a convolution $I^{\alpha} f=k_{\alpha} * f$ with the kernel

$$
k_{\alpha}(x)=\left\{\begin{array}{l}
\frac{2^{-\alpha} \Gamma((n-\alpha) / 2)}{\pi^{n / 2} \Gamma(\alpha / 2)}|x|^{\alpha-n} \quad \text { if } \alpha-n \neq 0,2,4, \ldots, \\
\frac{2^{1-\alpha}(-1)^{1+(\alpha-n) / 2}}{\pi^{n / 2} \Gamma(\alpha / 2)((\alpha-n) / 2) !}|x|^{\alpha-n} \log |x| \quad \text { if } a-n=0,2,4, \ldots .
\end{array}\right.
$$

The operator $I^{\alpha}$ is an automorphism of the space $\Phi\left(\mathrm{R}^{n}\right)$ and $\left(I^{\alpha} u\right)^{\wedge}(\xi)=|\xi|^{-\alpha} \hat{u}(\xi)$ for $u \in \Phi\left(\mathrm{R}^{n}\right)$. The last relation extends $I^{\alpha} u$ to all $\alpha \in \mathrm{C}$ as the entiere function of $\alpha$. The operator $D^{\alpha}=I^{-\alpha}, \operatorname{Re} \alpha>0$, is called the Riesz fractional derivative. For $f \in L^{p}\left(\mathrm{R}^{n}\right), 1 \leq p<n / \operatorname{Re} \alpha$, the integral $I^{\alpha} f$ exists a.e. on $\mathrm{R}^{n}$ and enjoys the Hardy-Littlewood-Sobolev 
theorem. In the case $p \geq n / \alpha$ the integral $I^{a} f$ can be divergent and the Riesz potential is defined as the $\Phi^{\prime}\left(\mathrm{R}^{n}\right)$-distribution: $\left(I^{\alpha} f, u\right)=\left(f, \overline{I^{\alpha}} \bar{u}\right), u \in \Phi\left(\mathrm{R}^{n}\right)$.

Lemma 2.5. Let $u \in \Phi\left(\mathrm{R}^{n}\right), \alpha \in C$. Then

$$
R^{\#} I^{\alpha-n+1} R u=2(2 \pi)^{n-1} I^{\alpha} u .
$$

For $u \in \mathcal{S}\left(\mathrm{R}^{n}\right)$ and real $\alpha<n$ this relation may found in [5], p. 18. In our case the proof is the same. The use of the space $\Phi\left(\mathrm{R}^{n}\right)$ enables us to avoid difficulties related to the fact that Riesz potentials do not preserve $\mathcal{S}\left(\mathrm{R}^{n}\right)$. By putting $\alpha=0$ and $\alpha=n-1$ in (2.5), in accordance with Lemma 2.2 we get

$$
u=\lambda_{n} R^{\#} D^{n-1} R u, \quad u=\lambda_{n} D^{n-1} R^{\#} R u, \quad R^{\#} R u=\lambda_{n}^{-1} I^{n-1} u, \quad u \in \Phi\left(\mathrm{R}^{n}\right),
$$

(2.7) $\omega=\lambda_{n} R D^{n-1} R^{\#} \omega, \quad \omega=\lambda_{n} D^{n-1} R R^{\#} \omega, \quad R R^{\#} \omega=\lambda_{n}^{-1} I^{n-1} \omega, \omega \in \Phi\left(\tilde{\mathrm{R}}^{n}\right)$, where $\lambda_{n}=2^{-1}(2 \pi)^{1-n}$ and abbreviations $D^{n-1}, I^{n-1}$ are used both for operators on $\mathrm{R}$ and for those on $\mathrm{R}^{n}$ (we hope the reader will not be confused).

\section{Inversion of the Radon transform (the first approach)}

Given a function $\varphi \in L^{1, r}\left(\tilde{\mathrm{R}}^{n}\right)$ and a finite Borel measure $\mu$ on $\mathrm{R}$, consider the truncated integral $T_{\varepsilon} \varphi$ (see (1.4)). By using the duality (2.4) and the continuity of the mapping $R: \mathcal{S}\left(\mathrm{R}^{n}\right) \rightarrow \mathcal{S}\left(\tilde{\mathrm{R}}^{n}\right)$, one can readily see that $T_{\varepsilon} \varphi$ is well-defined and belongs to $L_{l o c}^{1}\left(\mathrm{R}^{n}\right) \cap \mathcal{S}^{\prime}\left(\mathrm{R}^{n}\right)$.

Lemma 3.1. Let $\varphi=R f, f \in L^{p}\left(\mathrm{R}^{n}\right), 1 \leq p<n /(n-1)$. If $\mu$ satisfies the conditions of Theorem 1.1, then

$$
\left(T_{\varepsilon} \varphi\right)(x)=\left(f * h_{\varepsilon}\right)(x),
$$

$h_{\varepsilon}(x)=\varepsilon^{-n} h(x / \varepsilon), \quad h(x)=d_{n}|x|^{-n} \int_{-|x|}^{|x|}\left(|x|^{2}-s^{2}\right)^{(n-1) / 2} d \mu(s), \quad d_{n}=\frac{\pi^{(n-1) / 2}}{\Gamma((n+1) / 2)}$.

The function $h(x)$ enjoys the following properties:

(i) $h(x) \in L^{1}\left(\mathrm{R}^{n}\right), \quad h(x)=\left\{\begin{array}{l}O\left(|x|^{-1}\right) \text { if }|x|<1, \\ O\left(|x|^{-1-\gamma}\right), \gamma=\min (\beta, 2[(n-1) / 2]+2), \quad \text { if }|x|>1 ;\end{array}\right.$

(ii) $\int_{\mathrm{R}^{n}} h(x) d x=\left\{\begin{array}{l}\frac{\pi^{n+1 / 2}(-1)^{n / 2}}{\Gamma(n / 2) \Gamma((n+1) / 2)} \int_{-\infty}^{\infty}|s|^{n-1} d \mu(s) \quad \text { if } n \text { is even, } \\ \frac{2 \pi^{n-1 / 2}(-1)^{(n+1) / 2}}{\Gamma(n / 2) \Gamma((n+1) / 2)} \int_{-\infty}^{\infty}|s|^{n-1} \log |s| d \mu(s) \quad \text { if } n \text { is odd. }\end{array}\right.$ 
ProOF. By changing the order of integration, owing to the evenness of $R f$, we have

$$
\begin{aligned}
\left(T_{\varepsilon} R f\right)(x) & =\int_{-\infty}^{\infty} d \mu(s) \int_{\Sigma_{n-1}} d \theta \int_{\varepsilon}^{\infty}(R f)(\theta,\langle x, \theta\rangle-t s) \frac{d t}{t^{n}} \\
& =\int_{-\infty}^{\infty}|s|^{n-1} d \mu(s) \int_{\Sigma_{n-1}} d \theta \int_{\varepsilon|s|}^{\infty}(R f)(\theta,\langle x, \theta\rangle-\tau) \frac{d \tau}{\tau^{n}} \\
& =\int_{\Sigma_{n-1}} d \theta \int_{0}^{\infty}(R f)(\theta,\langle x, \theta\rangle-\tau) \frac{d \tau}{\tau^{n}} \int_{|s|<\tau / \varepsilon}|s|^{n-1} d \mu(s) \\
& =\frac{1}{2} \int_{\Sigma_{n-1}} d \theta \int_{-\infty}^{\infty}(R f)(\theta,\langle x, \theta\rangle-\tau) \frac{d \tau}{\tau^{n}} \int_{-|\tau| / \varepsilon}^{|\tau| / \varepsilon}|s|^{n-1} d \mu(s) \\
& =\frac{1}{2} \int_{\Sigma_{n-1}} d \theta \int_{\mathrm{R}^{n}} f(y) \frac{d y}{|\langle x-y, \theta\rangle|^{n}} \int_{-|\langle x-y, \theta\rangle| / \varepsilon}^{|\langle x-y\rangle| / \varepsilon}|s|^{n-1} d \mu(s)=\left(f * u_{\varepsilon}\right)(x)
\end{aligned}
$$

where $u_{\varepsilon}(x)=\varepsilon^{-n} u(x / \varepsilon)$,

$$
\begin{aligned}
u(x) & =\frac{1}{2} \int_{\Sigma_{n-1}} \frac{d \theta}{|\langle x, \theta\rangle|^{n}} \int_{|s|<|\langle x, \theta\rangle|}|s|^{n-1} d \mu(s)=\frac{\left|\Sigma_{n-2}\right|}{2|x|^{n}} \int_{|u|<1}\left(1-u^{2}\right)^{(n-3) / 2} \frac{d u}{|u|^{n}} \int_{|s|<|x||u|}|s|^{n-1} d \mu(s) \\
& =\frac{\left|\Sigma_{n-2}\right|}{|x|^{n}} \int_{|s|<|x|}|s|^{n-1} d \mu(s) \int_{|s| /|x|}^{1}\left(1-u^{2}\right)^{(n-3) / 2} \frac{d u}{u^{n}}=h(x) .
\end{aligned}
$$

The relations (i), (ii) follow from Lemma 2.1 of [9].

Proof of Theorem 1.1. The statement (i) (and therefore the implication (a) $\Rightarrow($ b)) follows from Lemma 3.1 due to the standard machinary of approximation to the identity. Let us prove $"(\mathrm{~b}) \Rightarrow(\mathrm{a})) "$. Assume that $T_{\varepsilon} \varphi$ converges in the $L^{p}$-norm to some function $f \in L^{p}\left(\mathrm{R}^{n}\right)$. Given a test function $\omega \in \Phi\left(\tilde{\mathrm{R}}^{n}\right)$, by taking into account the equality $R R^{\#} \omega=\lambda_{n}^{-1} I^{n-1} \omega$, $\lambda_{n}=2^{-1}(2 \pi)^{1-n}$, we have

$$
\begin{aligned}
(R f, \omega)^{\sim} & =\left(f, R^{\#} \omega\right)=\lim _{\varepsilon \rightarrow 0}\left(T_{\varepsilon} \varphi, R^{\#} \omega\right)=\lim _{\varepsilon \rightarrow 0} \int_{\varepsilon}^{\infty}\left(R^{\#}\left(\varphi * \mu_{t}\right), R^{\#} \omega\right) \frac{d t}{t^{n}} \\
& =\lim _{\varepsilon \rightarrow 0} \int_{\varepsilon}^{\infty}\left(\varphi * \mu_{t}, R R^{\#} \omega\right)^{\sim} \frac{d t}{t^{n}} \\
& =\lim _{\varepsilon \rightarrow 0} \lambda_{n}^{-1} \int_{\varepsilon}^{\infty}\left(\varphi * \mu_{t}, I^{n-1} \omega\right)^{\sim} \frac{d t}{t^{n}}=\lim _{\varepsilon \rightarrow 0} \lambda_{n}^{-1}\left(\varphi, \int_{\varepsilon}^{\infty} \frac{I^{n-1} \omega * \bar{\mu}_{t}}{t^{n}} d t\right)^{\sim} .
\end{aligned}
$$

According to Lemma 2.4 from [9], this yields $(R f, \omega)^{\sim}=\lim _{\varepsilon \rightarrow 0} \lambda_{n}^{-1}\left(\varphi, \omega * \bar{V}_{\varepsilon}\right)^{\sim}=\lim _{\varepsilon \rightarrow 0} \lambda_{n}^{-1}\left(\varphi * V_{\varepsilon}, \omega\right)^{\sim}$ where $V_{\varepsilon}(s)=\varepsilon^{-1} V(s / \varepsilon)$, $s \in \mathbf{R}^{1}$, 


$$
\begin{aligned}
& V(s)=\frac{2^{-n}}{\Gamma((n-1) / 2)} \int_{|s|^{2}}^{\infty}\left(\eta-|s|^{2}\right)^{(n-3) / 2} \lambda_{\mu, n-1}^{(1)}(\eta) \eta^{1-n / 2} d \eta, \\
& \lambda_{\mu, n-1}^{(1)}(\eta)=[\eta \Gamma((n+1) / 2)]^{-1} \int_{|y|^{2}<\eta}\left(\eta-|y|^{2}\right)^{(n-1) / 2} d \mu(y) .
\end{aligned}
$$

The function $V(s)$ belongs to $L^{1}(\mathrm{R})$, and $\int_{-\infty}^{\infty} V(s) d s=c_{1, \mu}$ where

$$
c_{1, \mu}=\left\{\begin{array}{l}
\frac{(-1)^{n / 2} 2^{-n} \pi^{3 / 2}}{\Gamma(n / 2) \Gamma((n+1) / 2)} \int_{-\infty}^{\infty}|s|^{n-1} d \mu(s) \quad \text { if } n \text { is even, } \\
\frac{(-1)^{(n+1) / 2} 2^{1-n} \pi^{1 / 2}}{\Gamma(n / 2) \Gamma((n+1) / 2)} \int_{-\infty}^{\infty}|s|^{n-1} \log |s| d \mu(s) \quad \text { if } n \text { is odd }
\end{array}\right.
$$

(see Lemma 2.2 from [9]). This gives $(R f, \omega)^{\sim}=c_{\mu}(\varphi, \omega)^{\sim}, c_{\mu}=c_{1, \mu} / \lambda_{n}$. Hence, by Lemma 2.5, $R f \stackrel{\text { a.e. }}{=} c_{\mu} \varphi$, which implies $(a)$.

The implication (a) $\Rightarrow$ (c) follows from (3.1). Let us prove "(c) $\Rightarrow$ (a)". According to (c), the set of functionals $\psi \rightarrow\left(T_{\varepsilon} \varphi, \psi\right), \psi \in L^{p^{\prime}}\left(\mathrm{R}^{n}\right)$, is bouded in $\left(L^{p^{\prime}}\right)^{*}$. Since the unit ball in the space, which is dual to the reflexive Banach space, is compact in the weak* topology, there exist a function $f \in L^{p}\left(\mathrm{R}^{n}\right)$ and a sequence $\varepsilon_{k} \rightarrow 0$ such that $\left(T_{\varepsilon_{k}} \varphi, \psi\right) \rightarrow(f, \psi)$ as $\varepsilon_{k} \rightarrow 0$ for all $\psi \in L^{p^{\prime}}\left(\mathrm{R}^{n}\right)$. For this $f$ and arbitrary $\omega \in \Phi\left(\tilde{R}^{n}\right)$ as above we have $(R f, \omega)^{\sim}=\left(f, R^{\#} \omega\right)=$ $\lim _{\varepsilon_{k} \rightarrow 0}\left(T_{\varepsilon_{k}} \varphi, R^{\#} \omega\right)=\lim _{\varepsilon_{k} \rightarrow 0} \lambda_{n}^{-1}\left(\varphi * V_{\varepsilon_{k}}, \omega\right)^{\sim}=c_{\mu}(\varphi, \omega)$, i.e. $c_{\mu} \varphi=R f$.

The described method can be used for inversion and characterization of Radon transforms of finite measures. Assume for simplicity that the wavelet measure $\mu \in \mathcal{M}(\mathrm{R})$ is absolutely continuous with the density $g(s) \in C_{0}(\mathrm{R}) \cap L^{1}(\mathrm{R})$. We recall (see Section 2) that, given a finite Borel measure $\lambda \in \mathcal{M}\left(\mathrm{R}^{n}\right)$, the Radon transform $R_{\theta} \lambda$ is a finite Borel measure on $\mathrm{R}$ (for each $\left.\theta \in \Sigma_{n-1}\right)$. Let $\mathcal{M}^{\#}\left(\tilde{\mathbf{R}}^{n}\right)$ be the set of all even finite Borel measures on $\tilde{\mathbf{R}}^{n}$. For $\theta \in \Sigma_{n-1}$ and $\sigma \in \mathcal{M}^{\#}\left(\tilde{\mathbf{R}}^{n}\right)$ we denote by $\sigma_{\theta}$ the image of $\sigma$ under the projection $(\theta, s) \rightarrow s$ "along the sphere" so that $\sigma_{\theta}(\Omega)=\sigma\left(\Sigma_{n-1} \times \Omega\right), \Omega \subset \mathbf{R}$. Clearly, $\sigma_{\theta}$ is a finite Borel measure on $\mathbf{R}$ (cf. [4], p. 16) and $\left\|\sigma_{\theta}\right\|=\|\sigma\|$. Given $\sigma \in \mathcal{M}^{\#}\left(\tilde{\mathrm{R}}^{n}\right)$ and $g(s) \in C_{0}(\mathrm{R}) \cap L^{1}(\mathrm{R})$, let

$$
T_{\varepsilon} \sigma=\int_{\varepsilon}^{\infty} R^{\#}\left(\sigma * g_{t}\right) \frac{d t}{t^{n}}, \quad \varepsilon>0 ; \quad\left(\sigma * g_{t}\right)(\theta, s)=\frac{1}{t} \int_{-\infty}^{\infty} g\left(\frac{s-\eta}{t}\right) d \sigma_{\theta}(\eta) .
$$

Since $\left\|\sigma * g_{t}\right\|_{1,1} \leq\|\sigma\|\|g\|_{1}$, then $R^{\#}\left(\sigma * g_{t}\right)$ is well-defined almost everywhere on $\mathrm{R}^{n}$ and $T_{\varepsilon} \sigma \in L_{\mathrm{loc}}^{1}\left(\mathrm{R}^{n}\right) \cap \mathcal{S}^{\prime}\left(\mathrm{R}^{n}\right)$.

THEOREM 3.2. Let $g \in C_{0}(\mathrm{R})$ satisfy the following conditions: 
$\int_{|s|>1}|s|^{\beta}|g(s)| d s<\infty$ for some $\beta>n-1 ; \int_{-\infty}^{\infty} s^{j} g(s) d s=0$ for all $j=0,1, \ldots, 2[(n-1) / 2]$.

(i) If $\sigma=R \lambda, \lambda \in \mathcal{M}\left(\mathrm{R}^{n}\right)$, then for each $u \in C_{0}\left(\mathrm{R}^{n}\right)$,

$$
\lim _{\varepsilon \rightarrow 0}\left(T_{\varepsilon} \sigma, u\right)=c_{g}(\lambda, u)
$$

where the constant $c_{g}$ is defined by (1.6) with $d \mu(s)=g(s) d s$.

(ii) If $c_{g} \neq 0$, then for $\sigma \in \mathcal{M}^{\#}\left(\tilde{\mathrm{R}}^{n}\right)$ the following statements are equivalent: (a) $\sigma \in R\left(\mathcal{M}\left(\mathrm{R}^{n}\right)\right)$; (b) the sequence $T_{\varepsilon} \sigma$ is weakly convergent as $\varepsilon \rightarrow 0$;

(c) $\sup _{\varepsilon>0}\left\|T_{\varepsilon} \sigma\right\|_{1}<\infty$.

Proof. (i) For $\psi \in C_{c}^{\infty}\left(\mathrm{R}^{n}\right)$ by (3.1) we have

$$
\begin{aligned}
\left(T_{\varepsilon} \sigma, \psi\right) & =\int_{\varepsilon}^{\infty}\left(\sigma * g_{t}, R \psi\right)^{\sim} \frac{d t}{t^{n}}=\left(\sigma, \int_{\varepsilon}^{\infty} \overline{R \bar{\psi} * g_{t}} \frac{d t}{t^{n}}\right)^{\sim} \\
& =\left(\lambda, \int_{\varepsilon}^{\infty} \overline{R^{\#}\left(R \bar{\psi} * g_{t}\right)} \frac{d t}{t^{n}}\right)=\left(\lambda, \overline{\bar{\psi} * h_{\varepsilon}}\right)=\left(\lambda * h_{\varepsilon}, \psi\right),
\end{aligned}
$$

where $h_{\varepsilon}$ is the same as in (3.1) with $d \mu(s)=g(s) d s$. Hence

$$
T_{\varepsilon} \sigma=\lambda * h_{\varepsilon},
$$

and the required result follows in an obvious way.

(ii) The implications (a) $\Rightarrow$ (b) and (a) $\Rightarrow$ (c) are implied by (3.4) and (3.5) respectively. If (b) holds, then, owing to the weak completeness of $\mathcal{M}\left(\mathrm{R}^{n}\right)$, there is a measure $\lambda \in \mathcal{M}\left(\mathrm{R}^{n}\right)$ such that $\left(T_{\varepsilon} \sigma, u\right)=(\lambda, u) \quad \forall u \in C_{0}\left(\mathrm{R}^{n}\right)$. Proceeding as in the proof of Theorem 1.1, for any $\omega \in \Phi\left(\tilde{R}^{n}\right)$ we have $(R \lambda, \omega)^{\sim}=\left(\lambda, R^{\#} \omega\right)=\lim \left(T_{\varepsilon} \sigma, R^{\#} \omega\right)=\left(c_{1, g} / \lambda_{n}\right)(\sigma, \omega)=c_{g}(\sigma, \omega)$. By Lemma 2.4(ii) it follows that ${ }^{\varepsilon} \vec{R} \lambda=c_{g} \sigma$ which gives (a). If (c) holds, then there is a sequence $\varepsilon_{k} \rightarrow 0$ and a measure $\lambda \in \mathcal{M}\left(\mathrm{R}^{n}\right)$ such that $\left(T_{\varepsilon_{k}} \sigma, u\right) \rightarrow(\lambda, u)$ as $\varepsilon_{k} \rightarrow 0$ for each $u \in C_{0}\left(\mathrm{R}^{n}\right)$. It remains to repeat the proof of " $(\mathrm{b}) \Rightarrow(\mathrm{a})$ " with $\varepsilon$ replaced by $\varepsilon_{k}$.

\section{The second approach}

In order to prove Theorem 1.4 we first represent the truncated integral $B_{\varepsilon} \varphi, \varphi=R f$, (see (1.14)) as the approximate identity.

Lemma 4.1. Assume that $\nu$ satisfies the conditions of Theorem 1.4. If $\varphi=R f, f \in L^{p}\left(\mathrm{R}^{n}\right), 1 \leq p<n /(n-1)$, then 


$$
B_{\varepsilon} \varphi=2(2 \pi)^{n-1} f * U_{\varepsilon},
$$

$U_{\varepsilon}(x)=\varepsilon^{-n} U(x / \varepsilon), \quad U(x)=\frac{2^{1-n}}{\Gamma((n-1) / 2)\left|\Sigma_{n-1}\right|} \int_{|x|^{2}}^{\infty}\left(\eta-|x|^{2}\right)^{(n-3) / 2} \lambda_{\nu, n-1}^{(n)}(\eta) \eta^{-n+3 / 2} d \eta$,

$$
\lambda_{\nu, n-1}^{(n)}(\eta)=[\eta \Gamma((n+1) / 2)]^{-1} \int_{|y|^{2}<\eta}\left(\eta-|y|^{2}\right)^{(n-1) / 2} d \nu(y) .
$$

The function $U(x)$ enjoys the following properties:

(i) $U(x) \in L^{1}\left(\mathrm{R}^{n}\right), \quad U(x)=\left\{\begin{array}{l}O\left(|x|^{-1}\right) \text { if }|x| \leq 1, \\ O\left(|x|^{-1-\gamma}\right), \gamma=\min (\beta, 2[(n-1) / 2]+2), \text { if }|x|>1 ;\end{array}\right.$

(ii) $c_{n, \nu}=\int_{\mathrm{R}^{n}} U(x) d x=$

$$
=\left\{\begin{array}{l}
\frac{2^{-n}(-1)^{n / 2} \pi \Gamma(n / 2)}{\Gamma(n-1 / 2) \Gamma((n+1) / 2)} \int_{\mathrm{R}^{n}}|y|^{n-1} d \nu(y) \quad \text { if } n \text { is even, } \\
\frac{2^{1-n}(-1)^{(n+1) / 2} \Gamma(n / 2)}{\Gamma(n-1 / 2) \Gamma((n+1) / 2)} \int_{\mathrm{R}^{n}}|y|^{n-1} \log |y| d \nu(y) \quad \text { if } n \text { is odd. }
\end{array}\right.
$$

Proof. The relations (i), (ii) follow from [9, Lemma 2.2]. Let us prove (4.1). Owing to the equality $R^{\#} R f=\lambda_{n}^{-1} I^{n-1} f, \lambda_{n}=(2 \pi)^{1-n} / 2$, by Lemma 2.4 from [9] for each $u \in \Phi\left(\mathrm{R}^{n}\right)$ we have

$$
\begin{aligned}
\left(B_{\varepsilon} \varphi, u\right) & \equiv\left(\int_{\mathrm{SO}(n)} d \gamma \int_{\varepsilon}^{\infty}\left(R^{\#} \varphi * \nu_{\gamma, t}\right) \frac{d t}{t^{n}}, u\right)=\left(\lambda_{n}^{-1} \int_{\mathrm{SO}(n)} d \gamma \int_{\varepsilon}^{\infty} \frac{I^{n-1} f * \nu_{\gamma, t}}{t^{n}} d t, u\right) \\
& =\lambda_{n}^{-1}\left(f, \int_{\mathrm{SO}(n)} d \gamma \int_{\varepsilon}^{\infty} \frac{I^{n-1} u * \bar{\nu}_{\gamma, t}}{t^{n}} d t\right)=\left(\lambda_{n}^{-1}\left(f, \bar{U}_{\varepsilon} * u\right)=\lambda_{n}^{-1}\left(f * U_{\varepsilon}, u\right) .\right.
\end{aligned}
$$

Since $U \in L^{1}\left(\mathrm{R}^{n}\right)$, then $f * U_{\varepsilon} \in L^{p}\left(\mathrm{R}^{n}\right)$. By the Hardy-Littlewood-Sobolev theorem, for $p>1$ we have $I^{n-1} f \in L^{q}\left(\mathrm{R}^{n}\right), q^{-1}=p^{-1}+n^{-1}-1$. If $p=1$, then $I^{n-1} f=c\left(g_{1}+g_{2}\right)$ where $g_{1}=\chi_{1}|x|^{-1} * f \in L^{p}\left(\mathrm{R}^{n}\right)$ and $g_{2}=\left(1-\chi_{1}\right)|x|^{-1} * f \in$ $L^{\rho}\left(\mathrm{R}^{n}\right), \rho^{-1}<p^{-1}+n^{-1}-1, \chi_{1}(x)$ being the characteristic function of the unit ball. The same relations hold for $B_{\varepsilon} \varphi$. By Lemma 2.3 it follows that $B_{\varepsilon} \varphi=\lambda_{n}^{-1} f * U_{\varepsilon}$ which coincides with (4.1).

Proof of Theorem 1.4. The statement (i) and the implication (a) $\Rightarrow$ (b) follow from Lemma 4.1. In order to prove "(b) $\Rightarrow(\mathrm{a})$ " we first remark that $\varphi=R f$ if and only if $R^{\#} \varphi=2(2 \pi)^{n-1} I^{n-1} f$ in the $\Phi^{\prime}\left(\mathrm{R}^{n}\right)$-sense. Indeed, let $\varphi=R f$, i.e. $(\varphi, \omega)^{\sim}=\left(f, R^{\#} \omega\right) \forall \omega \in \Phi\left(\tilde{R}^{n}\right)$. Then by (2.6) for $u \in \Phi\left(\mathbf{R}^{n}\right)$ we have $\left(R^{\#} \varphi, u\right)=(\varphi, R u)^{\sim}=\left(f, R^{\#} R u\right)=\lambda_{n}^{-1}\left(f, I^{n-1} u\right)=\lambda_{n}^{-1}\left(I^{n-1} f, u\right)$ where $\lambda_{n}^{-1}=2(2 \pi)^{n-1}$. Conversely, let $R^{\#} \varphi=\lambda_{n}^{-1} I^{n-1} f$ in the $\Phi^{\prime}\left(\mathrm{R}^{n}\right)$-sense. Then for each $u \in \Phi\left(\mathrm{R}^{n}\right)$ we get $\left(R^{\#} \varphi, u\right)=\lambda_{n}^{-1}\left(I^{n-1} f\right.$, u), i.e. $(\varphi, R u)^{\sim}=\lambda_{n}^{-1}\left(f, I^{n-1} u\right)$. 
Given a function $\omega \in \Phi\left(\tilde{\mathrm{R}}^{n}\right)$, put $\omega=R u_{0}, u_{0} \in \Phi\left(\mathrm{R}^{n}\right)$. Then $(\varphi, \omega)^{\sim}=\left(\varphi, R u_{0}\right)^{\sim}$ $=\lambda_{n}^{-1}\left(f, I^{n-1} u_{0}\right) \stackrel{(2.6)}{=}\left(f, R^{\#} \omega\right)=(R f, \omega)$. Since $R f \in L^{1, r}\left(\tilde{\mathrm{R}}^{n}\right)([6])$, then Lemma 2.4 yields $\varphi \stackrel{\text { a.e. }}{=} R f$.

Now let $\lim _{\varepsilon \rightarrow 0} B_{\varepsilon} \varphi=f$ in the $L^{p}\left(\mathrm{R}^{n}\right)$-norm. According to the remark from above it suffices to show that the function $g=R^{\#} \varphi$ is represented by the Riesz potential $I^{n-1} f$ in the $\Phi^{\prime}\left(\mathrm{R}^{n}\right)$-sense. Denote

$$
\stackrel{\circ}{D}_{\varepsilon}^{n-1} g=\int_{\mathrm{SO}(n)} d \gamma \int_{\varepsilon}^{\infty} \frac{g * \nu_{\gamma, t}}{t^{n}} d t \quad\left(=B_{\varepsilon} \varphi\right) .
$$

By making use of the relation

$$
\stackrel{\circ}{D}_{\varepsilon}^{n-1} I^{n-1} u \equiv \int_{\operatorname{SO}(n)} d \gamma \int_{\varepsilon}^{\infty} \frac{I^{n-1} u * \nu_{\gamma, t}}{t^{n}} d t=U_{\varepsilon} * u, \quad u \in \Phi\left(\mathrm{R}^{n}\right),
$$

(see Lemma 2.4 from [9]), for the arbitrary $u \in \Phi\left(\mathrm{R}^{n}\right)$ we obtain

$$
\left(I^{n-1} f, u\right)=\lim _{\varepsilon \rightarrow 0}\left(\stackrel{\circ}{D}_{\varepsilon}^{n-1} g, I^{n-1} u\right)=\lim _{\varepsilon \rightarrow 0}\left(g, \overline{\stackrel{\circ}{D}_{\varepsilon}^{n-1} I^{n-1} \bar{u}}\right)=\lim _{\varepsilon \rightarrow 0}\left(g, \overline{U_{\varepsilon} * \bar{u}}\right) .
$$

Let us show that $\lim _{\varepsilon \rightarrow 0}\left(g, \overline{U_{\varepsilon} * \bar{u}}\right)=c_{n, \nu}(g, u)$ where $c_{n, \nu}$ is defined by (4.2). Denote $\psi=R u$. Then $\Delta_{\varepsilon}=\left|\left(g, \overline{U_{\varepsilon} * \bar{u}}\right)-c_{n, \nu}(g, u)\right|=\left|\left(R^{\#} \varphi, \bar{U}_{\varepsilon} * u-\bar{c}_{n, \nu} u\right)\right|$ $=\left|\left(\varphi, R \bar{U}_{\varepsilon} * \psi-\bar{c}_{n, \nu} \psi\right)\right|$. We note that $R_{\theta} \bar{U}_{\varepsilon}=\left(R_{\theta} \bar{U}\right)_{\varepsilon}$ and $\int_{-\infty}^{\infty}\left(R_{\theta} \bar{U}\right)(s) d s=$ $\int_{\mathrm{R}^{n}} \bar{U}(x) d x \forall \theta \in \Sigma_{n-1}$. Hence $\lim _{\varepsilon \rightarrow 0} \Delta_{\varepsilon}=0$, i.e. $\left(I^{n-1} f, u\right)=c_{n, \nu}\left(R^{\#} \varphi, u\right)$ which was required.

The implication (a) $\Rightarrow(\mathrm{c})$ is clear from (4.1). Let us explain " (c) $\Rightarrow$ (a)". If $\sup _{\varepsilon>0}\left\|B_{\varepsilon} \varphi\right\|_{p}<\infty$, then for $g=R^{\#} \varphi$ we have $\sup _{\varepsilon>0}\left\|\stackrel{\circ}{D_{\varepsilon}^{n-1}} g\right\|_{p}<\infty$. Hence there exist $f \in L^{p}\left(\mathrm{R}^{n}\right)$ and a sequence $\varepsilon_{k} \rightarrow 0$ such that $\left(\stackrel{\circ}{D}_{\varepsilon}^{n-1} g, u\right) \rightarrow$ $(f, u) \forall u \in \Phi\left(\mathrm{R}^{n}\right)$. Proceeding as in the proof of "(b) $\Rightarrow$ (a)", we get $\left(I^{n-1} f, u\right)=c_{n, \nu}\left(R^{\#} \varphi, u\right)$ which implies (a).

In the framework of the method described in this section one can invert and characterize the Radon transforms of finite measures. Let $\nu$ in (1.15) be absolutely continuous with the rapidly decreasing continuous density $g(x)$ such that $\sup (1+|x|)^{k}|g(x)|<\infty, \forall k \in \mathrm{N}$. The collection of all such functions $g(x)$ will be denoted by $C_{\text {rap }}\left(\mathrm{R}^{n}\right)$. For an even finite Borel measure $\sigma$ on $\tilde{\mathrm{R}}^{n}$ (we recall the notation $\mathcal{M}^{\#}\left(\tilde{\mathrm{R}}^{n}\right)$ for this set) let

$$
B_{\varepsilon} \sigma=\int_{\mathrm{SO}(n)} d \gamma \int_{\varepsilon}^{\infty}\left(R^{\#} \sigma * g_{\gamma, t}\right) \frac{d t}{t^{n}}, \quad \varepsilon>0,
$$

where $R^{\#} \sigma$ is a tempered Borel measure on $\mathrm{R}^{n}$ (not necessarily finite) defined by the duality: $\left(R^{\#} \sigma, \omega\right)=(\sigma, R \omega)^{\sim}, \omega \in C_{c}\left(\mathrm{R}^{n}\right)$. The integral $B_{\varepsilon} \sigma$ is well-defined and belongs to $L_{\text {loc }}^{1}\left(\mathrm{R}^{n}\right) \cap \mathcal{S}^{\prime}\left(\mathrm{R}^{n}\right)$. 
TheOREM 4.2. Let $g \in C_{\text {rap }}\left(\mathrm{R}^{n}\right)$ be such that

$$
\int_{\mathrm{R}^{n}} x^{j} g(x) d x=0 \quad \text { for }|j|=0,2,4, \ldots, 2[(n-1) / 2] .
$$

(i) If $\sigma=R \lambda, \lambda \in \mathcal{M}\left(\mathrm{R}^{n}\right)$, then for each $\psi \in C_{0}\left(\mathrm{R}^{n}\right)$

$$
\lim _{\varepsilon \rightarrow 0}\left(B_{\varepsilon} \sigma, \psi\right)=d_{g}(\lambda, \psi)
$$

where $d_{g}$ is defined by (1.16) with $d \nu(y)=g(y) d y$.

(ii) If $d_{g} \neq 0$, then for $\sigma \in \mathcal{M}^{\#}\left(\tilde{\mathbf{R}}^{n}\right)$ the following statements are equivalent: (a) $\sigma \in R\left(\mathcal{M}\left(\mathrm{R}^{n}\right)\right)$; (b) the integral $B_{\varepsilon} \sigma$ weakly converges as $\varepsilon \rightarrow 0$;

(c) $\sup _{\varepsilon>0}\left\|B_{\varepsilon} \sigma\right\|_{1}<\infty$.

Proof. Denote $g_{\gamma, t}^{*}(x)=\bar{g}_{\gamma, t}(-x)$. For each $u \in C_{c}^{\infty}\left(\mathrm{R}^{n}\right)$ as in the proof of Lemma 4.1 we have

$$
\begin{aligned}
\left(B_{\varepsilon} \sigma, u\right) & =\int_{\mathrm{SO}(n)} d \gamma \int_{\varepsilon}^{\infty}\left(R^{\#} \sigma * g_{\gamma, t}, u\right) \frac{d t}{t^{n}}=\int_{\mathrm{SO}(n)} d \gamma \int_{\varepsilon}^{\infty}\left(\sigma, R\left(u * g_{\gamma, t}\right)\right)^{\sim} \frac{d t}{t^{n}} \\
& =\lambda_{n}^{-1}\left(\lambda, \int_{\mathrm{SO}(n)} d \gamma \int_{\varepsilon}^{\infty}\left(I^{n-1} u * g_{\gamma, t}^{*}\right) \frac{d t}{t^{n}}\right)=\lambda_{n}^{-1}\left(\lambda, \bar{U}_{\varepsilon} * u\right)=\lambda_{n}^{-1}\left(\lambda * U_{\varepsilon}, u\right) .
\end{aligned}
$$

Here the function $U(x)$ has the same form as in Lemma 4.1 with $d \nu(x)=g(x) d x$. Hence

$$
B_{\varepsilon} \sigma \stackrel{\text { a.e. }}{=} \lambda_{n}^{-1}\left(\lambda * U_{\varepsilon}\right),
$$

and the statement (i) (as well as the implication (a) $\Rightarrow$ (b)) follows.

In order to prove the inverse statement we note that given $\sigma \in \mathcal{M}^{\#}\left(\mathrm{R}^{n}\right)$ and $\lambda \in \mathcal{M}\left(\mathrm{R}^{n}\right)$, the equality $\sigma=R \lambda$ holds if and only if $R^{\#} \sigma=2(2 \pi)^{n-1} I^{n-1} \lambda$ in the $\Phi^{\prime}\left(\mathrm{R}^{n}\right)$-sense. This assertion is based on Lemma 2.4(ii) and can be checked in the same manner as the similar one in the proof of Theorem 1.4. Thus, it suffices to show that $d_{g}\left(R^{\#} \sigma, u\right)$ $=2(2 \pi)^{n-1}\left(\lambda, I^{n-1} u\right) \forall u \in \Phi\left(\mathrm{R}^{n}\right)$. By making use of (4.3) we have

$$
\left(\lambda, I^{n-1} u\right)=\lim _{\varepsilon \rightarrow 0}\left(B_{\varepsilon} \sigma, I^{n-1} u\right)=\lim _{\varepsilon \rightarrow 0}\left(R^{\#} \sigma, \psi_{\varepsilon}\right), \quad \psi_{\varepsilon}=\int_{\mathrm{SO}(n)} d \gamma \int_{\varepsilon}^{\infty} \frac{I^{n-1} u * \bar{g}_{\gamma, t}}{t^{n}} d t .
$$

Since $g \in C_{\text {rap }}\left(\mathrm{R}^{n}\right)$, then $\psi_{\varepsilon} \in C_{\text {rap }}\left(\mathrm{R}^{n}\right)$ and by (4.3), $\psi_{\varepsilon}=\bar{U}_{\varepsilon} * u$. Put $\omega=R u$. Then $\left(\lambda, I^{n-1} u\right)=\lim _{\varepsilon \rightarrow 0}\left(R^{\#} \sigma, \bar{U}_{\varepsilon} * u\right)=\lim _{\varepsilon \rightarrow 0}\left(\sigma, R \bar{U}_{\varepsilon} * \omega\right)^{\sim}$. Since $\sigma$ is finite, then due to the uniform estimate

$$
\left|\left(R \bar{U}_{\varepsilon} * \omega\right)(\theta, s)\right| \leq\|\omega\|_{\infty} \int_{-\infty}^{\infty}\left|\left(R_{\theta} \bar{U}_{\varepsilon}\right)(s)\right| d s \leq\|\omega\|_{\infty} \int_{\mathrm{R}^{n}}|U(x)| d x,
$$

one can write (cf. the proof of Theorem 1.4)

$$
\left(\lambda, I^{n-1} u\right)=\left(\sigma, \lim _{\varepsilon \rightarrow 0} R \bar{U}_{\varepsilon} * \omega\right)^{\sim}=c_{n, g}(\sigma, \omega)^{\sim}=c_{n, g}\left(R^{\#} \sigma, u\right)
$$


where $c_{n, g}$ has the form (4.2) with $\nu$ replaced by $g$. This yields the equality $d_{g}\left(R^{\#} \sigma, u\right)=2(2 \pi)^{n-1}\left(\lambda, I^{n-1} u\right)$, and the implication (b) $\Rightarrow$ (a) follows. The implication (a) $\Rightarrow$ (c) is clear from (4.7). If (c) holds, then there is a sequence $\varepsilon_{k} \rightarrow 0$ and a measure $\lambda \in \mathcal{M}\left(\mathrm{R}^{n}\right)$ such that $\left(B_{\varepsilon_{k}} \sigma, v\right) \rightarrow(\lambda, v)$ as $\varepsilon_{k} \rightarrow 0$ for all $v \in C_{0}\left(\mathrm{R}^{n}\right)$. Proceeding as above, for $u \in \Phi\left(\mathrm{R}^{n}\right)$ and $\omega=R u$ we have $\left(\lambda, I^{n-1} u\right)=\left(\sigma, \lim _{\varepsilon_{k} \rightarrow 0} R \bar{U}_{\varepsilon_{k}} * \omega\right)=c_{n, g}\left(R^{\#} \sigma, u\right)$ which implies (a).

\section{REFERENCES}

1. C. A. Berenstein and D. F. Walnut, Local inversion of the Radon transform in even dimensions using wavelets. In Proc. Conf. "75 Years of Radon Transform", Vienna, Austria 1992 (S.G. Gindikin, P. Michor, eds.), International Press, Hong Kong, 1994, 45-69.

2. S. Helgason, Geometric analysis on symmetric spaces, Amer. Math. Soc., Providence, RI, 1994.

3. M. Holschneider, Inverse Radon transforms through inverse wavelet transforms, Inverse Problems 7 (1991), 853-861.

4. P. Mattila, Geometry of Sets and Measures in Euclidean Spaces, Cambridge University Press, 1995.

5. F. Natterer, The Mathematics of Computerized Tomography, Wiley, New York, 1986.

6. D. M. Oberlin and E. M. Stein, Mapping properties of the Radon transform, Indiana Univ. Math. J. 31 (1982), 641-650.

7. B. Rubin, Fractional Integrals and Potentials, Addison Wesley Longman, Essex, U.K., 1996.

8. B. Rubin, The Calderón reproducing formula, windowed X-ray transforms and Radon transforms in $L^{p}$-spaces, The Journal of Fourier Analysis and Applications 4 (1998), 175-197.

9. B. Rubin, Wavelet type representations of Riesz fractional derivatives, The Hebrew University of Jerusalem, Preprint No. 12, 1995/96.

10. B. Rubin, Inversion and characterization of Radon transforms via continuous wavelet transforms. The Hebrew University of Jerusalem, Preprint No. 13, 1995/96.

11. S. G. Samko, A. A. Kilbas and O. I. Marichev, Integrals and derivatives of fractional order and some of their applications, Nauka i Teknika, Minsk, 1987 (Russian); English transl.: Fractional integrals and derivatives. Theory and applications, Gordon and Breach, London, 1993.

12. V. I. Semyanistyi, On some integral transformations in Euclidean space, Dokl. Akad. Nauk SSSR. 134 (1960), No. 3, 536-539 (Russian).

13. D. C. Solmon, A note on k-plane integral transforms, J. Math. Anal. Appl. 71 (1979), 351358.

14. D. Walnut, Applications of Gabor and wavelet expansions to the Radon transform, Probabilistic and Stochastic Methods in Analysis, with Applications, NATO ASI Series, J. Byrnes et al., eds., Kluwer Acad. Publ., The Netherlands, 1992, 187-205.

DEPARTMENT OF MATHEMATICS

THE HEBREW UNIVERSITY

91904, JERUSALEM

ISRAEL

Email: boris@math.huji.ac.il 